International Journal of Pure and Applied Mathematics

Volume 86 No. 4 2013, 727-746

ISSN: 1311-8080 (printed version); ISSN: 1314-3395 (on-line version)

url: http://www.ijpam.eu

doi: http://dx.doi.org/10.12732/ijpam.v86i4.11

\title{
NEW ESTIMATES ON GENERALIZATION OF SOME INTEGRAL INEQUALITIES FOR $s$-CONVEX FUNCTIONS AND THEIR APPLICATIONS
}

\author{
İmdat İşcan \\ Department of Mathematics \\ Faculty of Arts and Sciences \\ Giresun University \\ 28100, Giresun, TURKEY
}

\begin{abstract}
In this paper, a new identity for differentiable functions is derived. Thus we can obtain new estimates on generalization of Hadamard,Ostrowski and Simpson type inequalities for functions whose derivatives in absolute value at certain power are $s$-convex (in the second sense). Some applications to special means of real numbers are also given.
\end{abstract}

AMS Subject Classification: 26A51, 26D15

Key Words: Hermite-Hadamard inequality, Simpson type inequality, Ostrowski type inequality, trapezoid type inequality, midpoint type inequality, s-convex function

\section{Introduction}

Let $f: I \subseteq \mathbb{R} \rightarrow \mathbb{R}$ be a convex function defined on the interval $I$ of real numbers and $a, b \in I$ with $a<b$. The following inequality

$$
f\left(\frac{a+b}{2}\right) \leq \frac{1}{b-a} \int_{a}^{b} f(x) d x \leq \frac{f(a)+f(b)}{2} .
$$

holds. This double inequality is known in the literature as Hermite-Hadamard

Received: May 5, 2013

(c) 2013 Academic Publications, Ltd. url: www.acadpubl.eu 
integral inequality for convex functions [6]. Note that some of the classical inequalities for means can be derived from (1) for appropriate particular selections of the mapping $f$. Both inequalities hold in the reversed direction if $\mathrm{f}$ is concave.

Let $f: I \subseteq \mathbb{R} \rightarrow \mathbb{R}$ be a mapping differentiable in $I^{\circ}$, the interiorof $\mathrm{I}$, and let $a, b \in I^{\circ}$ with $a<b$. If $\left|f^{\prime}(x)\right| \leq M, x \in[a, b]$, then we the following inequality holds

$$
\left|f(x)-\frac{1}{b-a} \int_{a}^{b} f(t) d t\right| \leq \frac{M}{b-a}\left[\frac{(x-a)^{2}+(b-x)^{2}}{2}\right]
$$

for all $x \in[a, b]$. The constant $\frac{1}{4}$ is the best possible in the sense that it cannot be replaced by a smaller one. This result is known in the literature as the Ostrowski inequality [7].

The following inequality is well known in the literature as Simpson's inequality .

Let $f:[a, b] \rightarrow \mathbb{R}$ be a four times continuously differentiable mapping on $(a, b)$ and $\left\|f^{(4)}\right\|_{\infty}=\sup _{x \in(a, b)}\left|f^{(4)}(x)\right|<\infty$. Then the following inequality holds:

$$
\left|\frac{1}{3}\left[\frac{f(a)+f(b)}{2}+2 f\left(\frac{a+b}{2}\right)\right]-\frac{1}{b-a} \int_{a}^{b} f(x) d x\right| \leq \frac{1}{2880}\left\|f^{(4)}\right\|_{\infty}(b-a)^{2} .
$$

In recent years many authors have studied error estimations for Simpson's inequality; for refinements, counterparts, generalizations and new Simpson's type inequalities, see $[1,11,12,13]$ and therein.

In [4], Breckner introduced s-convex functions as a generalization of convex functions as follows:

Definition 1. Let $s \in(0,1]$ be a fixed real number. A function $f$ : $[0, \infty) \rightarrow[0, \infty)$ is said to be $s$-convex (in the second sense),or that $f$ belongs to the class $K_{s}^{2}$, if

$$
f(t x+(1-t) y) \leq t^{s} f(x)+(1-t)^{s} f(y)
$$

for all $x, y \in[0, \infty)$ and $t \in[0,1]$.

If inequality (2) is reversed, then $f$ is said to be $s$-concave (in the second sense). Of course, $s$-convexity means just convexity when $\mathrm{s}=1$.

In [5], Dragomir and Fitzpatrick proved a variant of Hadamard's inequality which holds for $s$-convex functions in the second sense. 
Theorem 2. Suppose that $f:[0, \infty) \rightarrow[0, \infty)$ is an $s$-convex function in the second sense, where $s \in(0,1)$, and let $a, b \in[0, \infty), a<b$.If $f \in L[a, b]$ then the following inequalities hold

$$
2^{s-1} f\left(\frac{a+b}{2}\right) \leq \frac{1}{b-a} \int_{a}^{b} f(x) d x \leq \frac{f(a)+f(b)}{s+1} .
$$

Both inequalities hold in the reversed direction if $\mathrm{f}$ is $s$-concave. The constant $k=\frac{1}{s+1}$ is the best possible in the second inequality in (3).

\section{Main Results}

In order to prove our main theorems, we need the following Lemma.

Lemma 3. Let $f: I \subseteq \mathbb{R} \rightarrow \mathbb{R}$ be a differentiable mapping on $I^{\circ}$ such that $f^{\prime} \in L[a, b]$, where $a, b \in I$ with $a<b$ and $\theta, \lambda \in[0,1]$. Then the following equality holds:

$$
\begin{aligned}
& (1-\theta)(\lambda f(a)+(1-\lambda) f(b))+\theta f((1-\lambda) a+\lambda b)-\frac{1}{b-a} \int_{a}^{b} f(x) d x \\
= & (b-a)\left[-\lambda^{2} \int_{0}^{1}(t-\theta) f^{\prime}(t a+(1-t)[(1-\lambda) a+\lambda b]) d t\right. \\
& \left.+(1-\lambda)^{2} \int_{0}^{1}(t-\theta) f^{\prime}(t b+(1-t)[(1-\lambda) a+\lambda b]) d t\right] .
\end{aligned}
$$

Proof. Firstly suppose that $\lambda \in(0,1)$ and let $C=(1-\lambda) a+\lambda b$.

$I=-\lambda^{2} \int_{0}^{1}(t-\theta) f^{\prime}(t a+(1-t) C) d t+(1-\lambda)^{2} \int_{0}^{1}(t-\theta) f^{\prime}(t b+(1-t) C) d t$

integrating by parts, we get

$$
I=\left.(t-\theta) \frac{\lambda f(t a+C(1-t))}{b-a}\right|_{0} ^{1}-\frac{\lambda}{b-a} \int_{0}^{1} f(t a+C(1-t)) d t
$$




$$
\begin{aligned}
& +\left.(t-\theta) \frac{(1-\lambda) f(t b+C(1-t))}{b-a}\right|_{0} ^{1}-\frac{(1-\lambda)}{b-a} \int_{0}^{1} f(t b+C(1-t)) d t \\
= & \lambda(1-\theta) \frac{f(a)}{b-a}+\lambda \theta \frac{f(C)}{b-a}+(1-\lambda)(1-\theta) \frac{f(b)}{b-a}+(1-\lambda) \theta \frac{f(C)}{b-a} \\
& -\frac{1}{b-a}\left[\lambda \int_{0}^{1} f(t a+C(1-t)) d t+(1-\lambda) \int_{0}^{1} f(t b+C(1-t)) d t\right]
\end{aligned}
$$

Setting $x=t a+C(1-t), d x=\lambda(a-b) d t$, and $x=t b+C(1-t), d x=$ $\mu(b-a) d t$ respectively, we obtain

$$
\begin{aligned}
& \lambda \int_{0}^{1} f(t a+C(1-t)) d t+(1-\lambda) \int_{0}^{1} f(t b+C(1-t)) d t \\
= & \frac{1}{b-a}\left[\int_{a}^{C} f(x) d x+\int_{C}^{b} f(x) d x=\frac{1}{b-a} \int_{a}^{b} f(x) d x\right.
\end{aligned}
$$

and so we have

$$
(b-a) I=(1-\theta)(\lambda f(a)+(1-\lambda) f(b))+\theta f(C)-\frac{1}{b-a} \int_{a}^{b} f(x) d x
$$

which gives the desired representation (4).

Secondly suppose that $\lambda \in\{0,1\}$. The identities

$$
(1-\theta) f(b)+\theta f(a)-\frac{1}{b-a} \int_{a}^{b} f(x) d x=(b-a) \int_{0}^{1}(t-\theta) f^{\prime}(t b+(1-t) a) d t
$$

and

$$
(1-\theta) f(a)+\theta f(b)-\frac{1}{b-a} \int_{a}^{b} f(x) d x=(b-a) \int_{0}^{1}(\theta-t) f^{\prime}(t a+(1-t) b) d t .
$$

can be proved by performing an integration by parts in the integrals from the right side and changing the variable. 
Theorem 4. Let $f: I \subseteq[0, \infty) \rightarrow \mathbb{R}$ be a differentiable mapping on $I^{\circ}$ such that $f^{\prime} \in L[a, b]$, where $a, b \in I^{\circ}$ with $a<b$ and $\theta, \lambda \in[0,1]$. If $\left|f^{\prime}\right|^{q}$ is $s$-convex on $[a, b]$, for some fixed $s \in(0,1]$ and $q \geq 1$, then the following inequality holds:

$$
\begin{gathered}
\left|(1-\theta)(\lambda f(a)+(1-\lambda) f(b))+\theta f((1-\lambda) a+\lambda b)-\frac{1}{b-a} \int_{a}^{b} f(x) d x\right| \\
\leq \quad(b-a) A_{1}^{1-\frac{1}{q}}(\theta)\left\{\lambda^{2}\left[\left|f^{\prime}(a)\right|^{q} A_{2}(\theta, s)+\left|f^{\prime}(C)\right|^{q} A_{3}(\theta, s)\right]^{\frac{1}{q}}\right. \\
\left.\quad+(1-\lambda)^{2}\left[\left|f^{\prime}(b)\right|^{q} A_{2}(\theta, s)+\left|f^{\prime}(C)\right|^{q} A_{3}(\theta, s)\right]^{\frac{1}{q}}\right\}
\end{gathered}
$$

where

$$
\begin{aligned}
A_{1}(\theta) & =\theta^{2}-\theta+\frac{1}{2} \\
A_{2}(\theta, s) & =\frac{2 \theta^{s+2}}{(s+1)(s+2)}-\frac{\theta}{s+1}+\frac{1}{s+2}, \\
A_{3}(\theta, s) & =\frac{2(1-\theta)^{s+2}}{(s+1)(s+2)}-\frac{1-\theta}{s+1}+\frac{1}{s+2} .
\end{aligned}
$$

and $C=(1-\lambda) a+\lambda b$.

Proof. Suppose that $q \geq 1$ and $C=(1-\lambda) a+\lambda b$. From Lemma 3 and using the well known power mean inequality, we have

$$
\begin{aligned}
& \left|(1-\theta)(\lambda f(a)+(1-\lambda) f(b))+\theta f(C)-\frac{1}{b-a} \int_{a}^{b} f(x) d x\right| \leq(b-a) \\
& {\left[\lambda^{2} \int_{0}^{1}|t-\theta|\left|f^{\prime}(t a+(1-t) C)\right| d t+(1-\lambda)^{2} \int_{0}^{1}|t-\theta|\left|f^{\prime}(t b+(1-t) C)\right| d t\right]} \\
& \leq(b-a)\left\{\lambda^{2}\left(\int_{0}^{1}|t-\theta| d t\right) \int_{0}^{1-\frac{1}{q}}|t-\theta| \mid f^{\prime}\left(t a+\left.(1-t) C\right|^{q} d t\right)^{\frac{1}{q}}\right. \\
& +(1-\lambda)^{2}\left(\int_{0}^{1}|t-\theta| d t\right)^{\frac{1}{q}} \int_{0}^{1}|t-\theta| \mid f^{\prime}\left(t b+\left.(1-t) C\right|^{q} d t\right\}^{1}
\end{aligned}
$$


Since $\left|f^{\prime}\right|^{q}$ is $s$-convex on $[a, b]$, we know that for $t \in[0,1]$

$$
\left|f^{\prime}(t a+C(1-t))\right|^{q} \leq t^{s}\left|f^{\prime}(a)\right|^{q}+(1-t)^{s}\left|f^{\prime}(C)\right|^{q}
$$

and

$$
\left|f^{\prime}(t b+C(1-t))\right|^{q} \leq t^{s}\left|f^{\prime}(b)\right|^{q}+(1-t)^{s}\left|f^{\prime}(C)\right|^{q} .
$$

Hence, by simple computation

$$
\begin{aligned}
& \int_{0}^{1}|t-\theta|\left|f^{\prime}(t a+(1-t) C)\right|^{q} d t \\
\leq & \int_{0}^{1}|t-\theta|\left[t^{s}\left|f^{\prime}(a)\right|^{q}+(1-t)^{s}\left|f^{\prime}(C)\right|^{q}\right] d t \\
= & \left|f^{\prime}(a)\right|^{q} \int_{0}^{1}|t-\theta| t^{s} d t+\left|f^{\prime}(C)\right|^{q}|t-\theta|(1-t)^{s} d t \\
= & \left|f^{\prime}(a)\right|^{q} A_{2}(\theta, s)+\left|f^{\prime}(C)\right|^{q} A_{3}(\theta, s) \\
\leq & \int_{0}^{1}|t-\theta|\left|f^{\prime}(t b+(1-t) C)\right|^{q} d t \\
\leq & \int_{0}^{1}|t-\theta|\left[t^{s}\left|f^{\prime}(b)\right|^{q}+(1-t)^{s}\left|f^{\prime}(C)\right|^{q}\right] d t \\
= & \left|f^{\prime}(b)\right|^{q} \int_{0}^{1}|t-\theta| t^{s} d t+\left|f^{\prime}(C)\right|^{q}|t-\theta|(1-t)^{s} d t \\
= & \left.\left|f_{0}^{\prime} A_{2}(\theta, s)+\right| f^{\prime}(C)\right|^{q} A_{3}(\theta, s)
\end{aligned}
$$

and

$$
\int_{0}^{1}|t-\theta| d t=\theta^{2}-\theta+\frac{1}{2} .
$$

Thus, using (8),(9) and (10) in (5), we obtain the inequality (4). This completes the proof. 
Corollary 5. Under the assumptions of Theorem 4 with $q=1$, we have

$$
\begin{gathered}
\left|(1-\theta)(\lambda f(a)+(1-\lambda) f(b))+\theta f((1-\lambda) a+\lambda b)-\frac{1}{b-a} \int_{a}^{b} f(x) d x\right| \\
\leq(b-a)\left\{A_{2}(\theta, s)\left(\lambda^{2}\left|f^{\prime}(a)\right|+(1-\lambda)^{2}\left|f^{\prime}(b)\right|\right)\right. \\
\left.+A_{3}(\theta, s)\left(2 \lambda^{2}-2 \lambda+1\right)\left|f^{\prime}(C)\right|\right\},
\end{gathered}
$$

where $A_{2}(\theta, s)$ and $A_{3}(\theta, s)$ are defined as in Theorem 4.

Corollary 6. Under the assumptions of Theorem 4 with $s=1$ we have

$$
\begin{aligned}
& \left|(1-\theta)(\lambda f(a)+(1-\lambda) f(b))+\theta f(C)-\frac{1}{b-a} \int_{a}^{b} f(x) d x\right| \\
& \leq \quad(b-a) A_{1}^{1-\frac{1}{q}}(\theta)\left\{\lambda^{2}\left[\left|f^{\prime}(a)\right|^{q} A_{2}(\theta, 1)+\left|f^{\prime}(C)\right|^{q} A_{3}(\theta, 1)\right]^{\frac{1}{q}}\right. \\
& \left.\quad+(1-\lambda)^{2}\left[\left|f^{\prime}(b)\right|^{q} A_{2}(\theta, 1)+\left|f^{\prime}(C)\right|^{q} A_{3}(\theta, 1)\right]^{\frac{1}{q}}\right\}
\end{aligned}
$$

where

$$
\begin{aligned}
A_{1}(\theta) & =\theta^{2}-\theta+\frac{1}{2} \\
A_{2}(\theta, 1) & =\frac{1+\theta^{3}}{3}-\frac{\theta}{2}, \\
A_{3}(\theta, 1) & =\frac{1+(1-\theta)^{3}}{3}-\frac{1-\theta}{2},
\end{aligned}
$$

and $C=(1-\lambda) a+\lambda b$.

Corollary 7. Under the assumptions of Theorem 4 with $\theta=1$, then we have following generalized midpoint type inequality

$$
\begin{aligned}
& \left|f((1-\lambda) a+\lambda b)-\frac{1}{b-a} \int_{a}^{b} f(x) d x\right| \\
\leq & \frac{b-a}{2}\left(\frac{2}{(s+1)(s+2)}\right)^{\frac{1}{q}}\left\{\lambda^{2}\left(\left|f^{\prime}(a)\right|^{q}+(s+1)\left|f^{\prime}(C)\right|^{q}\right)^{\frac{1}{q}}\right. \\
& \left.+(1-\lambda)^{2}\left(\left|f^{\prime}(b)\right|^{q}+(s+1)\left|f^{\prime}(C)\right|^{q}\right)^{\frac{1}{q}}\right\},
\end{aligned}
$$

where $C=(1-\lambda) a+\lambda b$. 
Corollary 8. Under the assumptions of Theorem 4 with $\theta=1$, if $\left|f^{\prime}(x)\right| \leq$ $M, x \in[a, b]$, then we have the following Ostrowski type inequality

$$
\left|f(x)-\frac{1}{b-a} \int_{a}^{b} f(u) d u\right| \leq M\left(\frac{2}{s+1}\right)^{\frac{1}{q}}\left[\frac{(x-a)^{2}+(b-x)^{2}}{2(b-a)}\right]
$$

for each $x \in[a, b]$.

Proof. For each $x \in[a, b]$, there exist $\lambda_{x} \in[0,1]$ such that $x=\left(1-\lambda_{x}\right) a+$ $\lambda_{x} b$. Hence we have $\lambda_{x}=\frac{x-a}{b-a}$ and $1-\lambda_{x}=\frac{b-x}{b-a}$. Therefore for each $x \in[a, b]$, from the inequality (4) we obtain the inequality (11).

Remark 1. We note that the inequality (11) is the same of the inequality in [2, Theorem 4].

Corollary 9. Under the assumptions of Theorem 4 with $\theta=0$, then we have following generalized trapezoid type inequality

$$
\begin{aligned}
& \left|\lambda f(a)+(1-\lambda) f(b)-\frac{1}{b-a} \int_{a}^{b} f(x) d x\right| \\
\leq & \frac{b-a}{2}\left(\frac{2}{(s+1)(s+2)}\right)^{\frac{1}{q}}\left\{\lambda^{2}\left((s+1)\left|f^{\prime}(a)\right|^{q}+\left|f^{\prime}(C)\right|^{q}\right)^{\frac{1}{q}}\right. \\
& \left.+(1-\lambda)^{2}\left((s+1)\left|f^{\prime}(b)\right|^{q}+\left|f^{\prime}(C)\right|^{q}\right)^{\frac{1}{q}}\right\},
\end{aligned}
$$

where $C=(1-\lambda) a+\lambda b$.

Corollary 10. Under the assumptions of Theorem 4 with $\lambda=\frac{1}{2}$ and $\theta=\frac{2}{3}$, then we have the following Simpson type inequality

$$
\begin{aligned}
& \left|\frac{1}{6}\left[f(a)+4 f\left(\frac{a+b}{2}\right)+f(b)\right]-\frac{1}{b-a} \int_{a}^{b} f(x) d x\right| \\
\leq & \frac{b-a}{4}\left(\frac{5}{18}\right)^{1-\frac{1}{q}}\left\{\left(A_{2}\left(\frac{2}{3}, s\right)\left|f^{\prime}(a)\right|^{q}+A_{3}\left(\frac{2}{3}, s\right)\left|f^{\prime}\left(\frac{a+b}{2}\right)\right|^{q}\right)^{\frac{1}{q}}\right. \\
& \left.+\left(A_{2}\left(\frac{2}{3}, s\right)\left|f^{\prime}(b)\right|^{q}+A_{3}\left(\frac{2}{3}, s\right)\left|f^{\prime}\left(\frac{a+b}{2}\right)\right|^{q}\right)^{\frac{1}{q}}\right\}
\end{aligned}
$$


where

$$
\begin{aligned}
& A_{2}\left(\frac{2}{3}, s\right)=\frac{2^{s+3}+3^{s+1}(s-1)}{3^{s+2}(s+1)(s+2)} \\
& A_{3}\left(\frac{2}{3}, s\right)=\frac{2+3^{s+1}(2 s+1)}{3^{s+2}(s+1)(s+2)} .
\end{aligned}
$$

Corollary 11. Under the assumptions of Theorem 4 with $\lambda=\frac{1}{2}$ and $\theta=1$, then we have following midpoint type inequality

$$
\begin{aligned}
& \left|f\left(\frac{a+b}{2}\right)-\frac{1}{b-a} \int_{a}^{b} f(x) d x\right| \leq \frac{b-a}{8}\left(\frac{2}{(s+1)(s+2)}\right)^{\frac{1}{q}} \\
& \times\left\{\left(\left|f^{\prime}(a)\right|^{q}+(s+1)\left|f^{\prime}\left(\frac{a+b}{2}\right)\right|^{q}\right)^{\frac{1}{q}}\right. \\
& \left.+\left(\left|f^{\prime}(b)\right|^{q}+(s+1)\left|f^{\prime}\left(\frac{a+b}{2}\right)\right|^{q}\right)^{\frac{1}{q}}\right\} .
\end{aligned}
$$

Corollary 12. Under the assumptions of Theorem 4 with $\lambda=\frac{1}{2}$, and $\theta=0$, then we get the following trapezoid type inequality

$$
\begin{aligned}
& \left|\frac{f(a)+f(b)}{2}-\frac{1}{b-a} \int_{a}^{b} f(x) d x\right| \leq \frac{b-a}{8}\left(\frac{2}{(s+1)(s+2)}\right)^{\frac{1}{q}} \\
& \times\left\{\left((s+1)\left|f^{\prime}(a)\right|^{q}+\left|f^{\prime}\left(\frac{a+b}{2}\right)\right|^{q}\right)^{\frac{1}{q}}\right. \\
& \left.+\left((s+1)\left|f^{\prime}(b)\right|^{q}+\left|f^{\prime}\left(\frac{a+b}{2}\right)\right|^{q}\right)^{\frac{1}{q}}\right\} .
\end{aligned}
$$

Using Lemma 3 we shall give another result for $s$-convex functions as follows.

Theorem 13. Let $f: I \subseteq[0, \infty) \rightarrow \mathbb{R}$ be a differentiable mapping on $I^{\circ}$ such that $f^{\prime} \in L[a, b]$, where $a, b \in I^{\circ}$ with $a<b$ and $\theta, \lambda \in[0,1]$. If $\left|f^{\prime}\right|^{q}$ is $s$-convex on $[a, b]$, for some fixed $s \in(0,1]$ and $q>1$, then the following inequality holds:

$$
\left|(1-\theta)(\lambda f(a)+(1-\lambda) f(b))+\theta f((1-\lambda) a+\lambda b)-\frac{1}{b-a} \int_{a}^{b} f(x) d x\right|
$$




$$
\begin{aligned}
\leq & (b-a)\left(\frac{\theta^{p+1}+(1-\theta)^{p+1}}{p+1}\right)^{\frac{1}{p}} \\
& \times\left[\lambda^{2}\left(\frac{\left|f^{\prime}(a)\right|^{q}+\left|f^{\prime}(C)\right|^{q}}{s+1}\right)^{\frac{1}{q}}+(1-\lambda)^{2}\left(\frac{\left|f^{\prime}(b)\right|^{q}+\left|f^{\prime}(C)\right|^{q}}{s+1}\right)^{\frac{1}{q}}\right] .
\end{aligned}
$$

where $C=(1-\lambda) a+\lambda b$ and $\frac{1}{p}+\frac{1}{q}=1$.

Proof. Suppose that $C=(1-\lambda) a+\lambda b$. From Lemma 3 and by Hölder's integral inequality, we have

$$
\begin{gathered}
\left|(1-\theta)(\lambda f(a)+(1-\lambda) f(b))+\theta f(C)-\frac{1}{b-a} \int_{a}^{b} f(x) d x\right| \leq(b-a) \\
{\left[\lambda^{2} \int_{0}^{1}|t-\theta|\left|f^{\prime}(t a+(1-t) C)\right| d t+(1-\lambda)^{2} \int_{0}^{1}|t-\theta|\left|f^{\prime}(t b+(1-t) C)\right| d t\right]} \\
\leq(b-a)\left\{\lambda^{2}\left(\int_{0}^{1}|t-\theta|^{p} d t \int^{\frac{1}{p}}\left(\int_{0}^{1} \mid f^{\prime}(t a+(1-t) C)^{q} d t\right)^{\frac{1}{q}}\right)^{\frac{1}{q}}\right\}^{\frac{1}{p}}\left(\int_{0}^{1}\left|f^{\prime}(t b+(1-t) C)\right|^{q} d t\right)_{0}^{1}|t-\theta|^{p} d t
\end{gathered}
$$

Since $\left|f^{\prime}\right|^{q}$ is $s$-convex on $[a, b]$, the inequalities (6) and (7) holds. Hence, by simple computation

$$
\begin{aligned}
\int_{0}^{1}\left|f^{\prime}(t a+(1-t) C)\right|^{q} d t & \leq \int_{0}^{1} t^{s}\left|f^{\prime}(a)\right|^{q}+(1-t)^{s}\left|f^{\prime}(C)\right|^{q} \\
& =\frac{\left|f^{\prime}(a)\right|^{q}+\left|f^{\prime}(C)\right|^{q}}{s+1} \\
\int_{0}^{1}\left|f^{\prime}(t b+(1-t) C)\right|^{q} d t & \leq \int_{0}^{1} t^{s}\left|f^{\prime}(b)\right|^{q}+(1-t)^{s}\left|f^{\prime}(C)\right|^{q} \\
& =\frac{\left|f^{\prime}(b)\right|^{q}+\left|f^{\prime}(C)\right|^{q}}{s+1}
\end{aligned}
$$


and

$$
\int_{0}^{1}|t-\theta|^{p} d t=\frac{\theta^{p+1}+(1-\theta)^{p+1}}{p+1}
$$

thus, using (14)-(16) in (13), we obtain the inequality (12). This completes the proof.

Corollary 14. Under the assumptions of Theorem 13 with $s=1$, we have

$$
\begin{aligned}
& \left|(1-\theta)(\lambda f(a)+(1-\lambda) f(b))+\theta f((1-\lambda) a+\lambda b)-\frac{1}{b-a} \int_{a}^{b} f(x) d x\right| \\
& \leq \quad(b-a)\left(\frac{\theta^{p+1}+(1-\theta)^{p+1}}{p+1}\right)^{\frac{1}{p}}\left(\frac{1}{2}\right)^{\frac{1}{q}} \\
& \quad \times\left[\lambda^{2}\left(\left|f^{\prime}(a)\right|^{q}+\left|f^{\prime}(C)\right|^{q}\right)^{\frac{1}{q}}+(1-\lambda)^{2}\left(\left|f^{\prime}(b)\right|^{q}+\left|f^{\prime}(C)\right|^{q}\right)^{\frac{1}{q}}\right],
\end{aligned}
$$

where $C=(1-\lambda) a+\lambda b$ and $\frac{1}{p}+\frac{1}{q}=1$.

Corollary 15. Under the assumptions of Theorem 13 with $\theta=1$, then we have the following generalized midpoint type inequality

$$
\begin{aligned}
\mid f((1- & \lambda) a+\lambda b)-\frac{1}{b-a} \int_{a}^{b} f(x) d x \mid \leq(b-a)\left(\frac{1}{p+1}\right)^{\frac{1}{p}} \\
\times & {\left[\lambda^{2}\left(\frac{\left|f^{\prime}(a)\right|^{q}+\left|f^{\prime}(C)\right|^{q}}{s+1}\right)^{\frac{1}{q}}+(1-\lambda)^{2}\left(\frac{\left|f^{\prime}(b)\right|^{q}+\left|f^{\prime}(C)\right|^{q}}{s+1}\right)^{\frac{1}{q}}\right], }
\end{aligned}
$$

where $C=(1-\lambda) a+\lambda b$ and $\frac{1}{p}+\frac{1}{q}=1$.

Corollary 16. Under the assumptions of Theorem 13 with $\theta=0$, then we have the following generalized trapezoid type inequality

$$
\begin{aligned}
\mid \lambda f(a) & +(1-\lambda) f(b)-\frac{1}{b-a} \int_{a}^{b} f(x) d x \mid \leq(b-a)\left(\frac{1}{p+1}\right)^{\frac{1}{p}} \\
& \times\left[\lambda^{2}\left(\frac{\left|f^{\prime}(a)\right|^{q}+\left|f^{\prime}(C)\right|^{q}}{s+1}\right)^{\frac{1}{q}}+(1-\lambda)^{2}\left(\frac{\left|f^{\prime}(b)\right|^{q}+\left|f^{\prime}(C)\right|^{q}}{s+1}\right)^{\frac{1}{q}}\right],
\end{aligned}
$$

where $C=(1-\lambda) a+\lambda b$ and $\frac{1}{p}+\frac{1}{q}=1$. 
Corollary 17. Under the assumptions of Theorem 13 with $\theta=1$, if $\left|f^{\prime}(x)\right| \leq M, x \in[a, b]$, then we have the following Ostrowski type inequality

$$
\left|f(x)-\frac{1}{b-a} \int_{a}^{b} f(u) d u\right| \leq \frac{M}{(p+1)^{\frac{1}{p}}}\left(\frac{2}{s+1}\right)^{\frac{1}{q}}\left[\frac{(x-a)^{2}+(b-x)^{2}}{b-a}\right]
$$

for each $x \in[a, b]$.

Proof. For each $x \in[a, b]$, there exist $\lambda_{x} \in[0,1]$ such that $x=\left(1-\lambda_{x}\right) a+$ $\lambda_{x} b$. Hence we have $\lambda_{x}=\frac{x-a}{b-a}$ and $1-\lambda_{x}=\frac{b-x}{b-a}$. Therefore for each $x \in[a, b]$, from the inequality (12) we obtain the inequality (17).

Remark 2. We note that the inequality (17) is the same of the inequality in [2, Theorem 3].

Corollary 18. Under the assumptions of Theorem 13 with $\lambda=\frac{1}{2}$ and $\theta=\frac{2}{3}$, then we have the following Simpson type inequality

$$
\begin{aligned}
& \left|\frac{1}{6}\left[f(a)+4 f\left(\frac{a+b}{2}\right)+f(b)\right]-\frac{1}{b-a} \int_{a}^{b} f(x) d x\right| \\
& \leq \frac{b-a}{12}\left(\frac{1+2^{p+1}}{3(p+1)}\right)^{\frac{1}{p}} \\
& \quad \times\left\{\left(\frac{\left|f^{\prime}\left(\frac{a+b}{2}\right)\right|^{q}+\left|f^{\prime}(a)\right|^{q}}{s+1}\right)^{\frac{1}{q}}+\left(\frac{\left|f^{\prime}\left(\frac{a+b}{2}\right)\right|^{q}+\left|f^{\prime}(b)\right|^{q}}{s+1}\right)^{\frac{1}{q}}\right\},
\end{aligned}
$$

which is the same of the inequality in [12, Theorem 8].

Corollary 19. Under the assumptions of Theorem 13 with $\lambda=\frac{1}{2}$ and $\theta=1$, then we have the following midpoint type inequality

$$
\begin{aligned}
& \left|f\left(\frac{a+b}{2}\right)-\frac{1}{b-a} \int_{a}^{b} f(x) d x\right| \leq \frac{b-a}{4}\left(\frac{1}{p+1}\right)^{\frac{1}{p}} \\
& \times\left\{\left(\frac{\left|f^{\prime}\left(\frac{a+b}{2}\right)\right|^{q}+\left|f^{\prime}(a)\right|^{q}}{s+1}\right)^{\frac{1}{q}}+\left(\frac{\left|f^{\prime}\left(\frac{a+b}{2}\right)\right|^{q}+\left|f^{\prime}(b)\right|^{q}}{s+1}\right)^{\frac{1}{q}}\right\} .
\end{aligned}
$$


Remark 3. We note that the inequality (18) is better than the inequality in [3, Theorem 2.3]. Because, by inequality

$$
2^{s-1}\left|f^{\prime}\left(\frac{a+b}{2}\right)\right|^{q} \leq \frac{\left|f^{\prime}(a)\right|^{q}+\left|f^{\prime}(b)\right|^{q}}{s+1}
$$

we have

$$
\begin{aligned}
& \mid f\left(\frac{a+b}{2}\right)- \frac{1}{b-a} \int_{a}^{b} f(x) d x \mid \leq\left(\frac{b-a}{4}\right)\left(\frac{1}{p+1}\right)^{\frac{1}{p}}\left(\frac{1}{s+1}\right)^{\frac{2}{q}} \\
& \times\left[\left(\left(2^{1-s}+s+1\right)\left|f^{\prime}(a)\right|^{q}+2^{1-s}\left|f^{\prime}(b)\right|^{q}\right)^{\frac{1}{q}}\right. \\
&\left.+\left(\left(2^{1-s}+s+1\right)\left|f^{\prime}(b)\right|^{q}+2^{1-s}\left|f^{\prime}(a)\right|^{q}\right)^{\frac{1}{q}}\right]
\end{aligned}
$$

which is the same of the inequality in [3, Theorem 2.3].

Corollary 20. Under the assumptions of Theorem 13 with $\lambda=\frac{1}{2}$ and $\theta=0$, then we have the following trapezoid type inequality

$$
\begin{aligned}
& \left|\frac{f(a)+f(b)}{2}-\frac{1}{b-a} \int_{a}^{b} f(x) d x\right| \leq \frac{b-a}{4}\left(\frac{1}{p+1}\right)^{\frac{1}{p}} \\
& \times\left\{\left(\frac{\left|f^{\prime}\left(\frac{a+b}{2}\right)\right|^{q}+\left|f^{\prime}(a)\right|^{q}}{s+1}\right)^{\frac{1}{q}}+\left(\frac{\left|f^{\prime}\left(\frac{a+b}{2}\right)\right|^{q}+\left|f^{\prime}(b)\right|^{q}}{s+1}\right)^{\frac{1}{q}}\right\} .
\end{aligned}
$$

We note that the obtained inequality (19) is better than the first inequality in [9, Theorem 3].

Theorem 21. Let $f: I \subseteq[0, \infty) \rightarrow \mathbb{R}$ be a differentiable mapping on $I^{\circ}$ such that $f^{\prime} \in L[a, b]$, where $a, b \in I^{\circ}$ with $a<b$ and $\alpha, \lambda \in[0,1]$. If $\left|f^{\prime}\right|^{q}$ is $s$-concave on $[a, b]$, for some fixed $s \in(0,1]$ and $q>1$, then the following inequality holds:

$$
\begin{aligned}
& \left|(1-\theta)(\lambda f(a)+(1-\lambda) f(b))+\theta f((1-\lambda) a+\lambda b)-\frac{1}{b-a} \int_{a}^{b} f(x) d x\right| \\
& \quad \leq(b-a)\left(\frac{1}{2}\right)^{\frac{1-s}{q}}\left(\frac{\theta^{p+1}+(1-\theta)^{p+1}}{p+1}\right)^{\frac{1}{p}}
\end{aligned}
$$




$$
\times\left\{\lambda^{2}\left|f^{\prime}\left(\frac{(2-\lambda) a+\lambda b}{2}\right)\right|+(1-\lambda)^{2}\left|f^{\prime}\left(\frac{(1-\lambda) a+(1+\lambda) b}{2}\right)\right|\right\},
$$

where $C=(1-\lambda) a+\lambda b$ and $1 / p+1 / q=1$.

Proof. Suppose that $C=(1-\lambda) a+\lambda b$. We proceed similarly as in the proof Theorem 13. Since $\left|f^{\prime}\right|^{q}$ is $s$-concave on $[a, b], \lambda \in(0,1]$ by the inequality (3), we get

$$
\begin{aligned}
& \frac{1}{C-a} \int_{a}^{C}\left|f^{\prime}(x)\right|^{q} d x \\
= & \int_{0}^{1}\left|f^{\prime}(t a+(1-t) C)\right|^{q} d t \leq \frac{1}{2^{1-s}}\left|f^{\prime}\left(\frac{(2-\lambda) a+\lambda b}{2}\right)\right|^{q}
\end{aligned}
$$

the inequality (21) also holds $\lambda=0$. Similarly, for $\lambda \in[0,1)$ by the inequality (3), we have

$$
\begin{aligned}
& \frac{1}{b-C} \int_{C}^{b}\left|f^{\prime}(x)\right|^{q} d x \\
= & \int_{0}^{1}\left|f^{\prime}(t b+(1-t) C)\right|^{q} d t \\
\leq & \frac{1}{2^{1-s}}\left|f^{\prime}\left(\frac{(1-\lambda) a+(1+\lambda) b}{2}\right)\right|^{q}
\end{aligned}
$$

the inequality (22) also holds $\lambda=1$. Thus using (16), (21) and (22) in (13), we obtain the inequality (20). This completes the proof.

Corollary 22. Under the assumptions of Theorem 21 with $s=1$, we have

$$
\begin{aligned}
& \left|(1-\theta)(\lambda f(a)+(1-\lambda) f(b))+\theta f((1-\lambda) a+\lambda b)-\frac{1}{b-a} \int_{a}^{b} f(x) d x\right| \\
\leq & (b-a)\left(\frac{\theta^{p+1}+(1-\theta)^{p+1}}{p+1}\right)^{\frac{1}{p}} \\
& \times\left\{\lambda^{2}\left|f^{\prime}\left(\frac{(2-\lambda) a+\lambda b}{2}\right)\right|+(1-\lambda)^{2}\left|f^{\prime}\left(\frac{(1-\lambda) a+(1+\lambda) b}{2}\right)\right|\right\} .
\end{aligned}
$$


Remark 4. In Corollary 22, if we take $\lambda=\frac{1}{2}$ and $\theta=0$, then we have the following trapezoid type inequality.

$$
\begin{aligned}
& \left|\frac{f(a)+f(b)}{2}-\frac{1}{b-a} \int_{a}^{b} f(x) d x\right| \\
\leq & \frac{b-a}{4}\left(\frac{1}{p+1}\right)^{\frac{1}{p}}\left[\left|f^{\prime}\left(\frac{3 b+a}{4}\right)\right|+\left|f^{\prime}\left(\frac{3 a+b}{4}\right)\right|\right]
\end{aligned}
$$

which is the same of the inequality in [9, Theorem 2]

Remark 5. In Corollary 22, if we take $\lambda=\frac{1}{2}$ and $\theta=1$, then we have the following midpoint type inequality

$$
\begin{aligned}
& \left|f\left(\frac{a+b}{2}\right)-\frac{1}{b-a} \int_{a}^{b} f(x) d x\right| \\
\leq & \frac{b-a}{4}\left(\frac{1}{p+1}\right)^{\frac{1}{p}}\left[\left|f^{\prime}\left(\frac{3 b+a}{4}\right)\right|+\left|f^{\prime}\left(\frac{3 a+b}{4}\right)\right|\right]
\end{aligned}
$$

which is the same of the inequality in [3, Theorem 2.5].

Corollary 23. Under the assumptions of Theorem 21 with $\theta=0$, we have the following generalized trapezoid type inequality

$$
\begin{aligned}
& \left|\lambda f(a)+(1-\lambda) f(b)-\frac{1}{b-a} \int_{a}^{b} f(x) d x\right| \\
\leq & (b-a)\left(\frac{1}{2}\right)^{\frac{1-s}{q}}\left(\frac{1}{p+1}\right)^{\frac{1}{p}} \\
& \times\left\{\lambda^{2}\left|f^{\prime}\left(\frac{(2-\lambda) a+\lambda b}{2}\right)\right|+(1-\lambda)^{2}\left|f^{\prime}\left(\frac{(1-\lambda) a+(1+\lambda) b}{2}\right)\right|\right\},
\end{aligned}
$$

Corollary 24. Under the assumptions of Theorem 21 with $\theta=1$, we have the following generalized midpoint type inequality

$$
\begin{aligned}
& \left|f((1-\lambda) a+\lambda b)-\frac{1}{b-a} \int_{a}^{b} f(x) d x\right| \\
\leq & (b-a)\left(\frac{1}{2}\right)^{\frac{1-s}{q}}\left(\frac{1}{p+1}\right)^{\frac{1}{p}}
\end{aligned}
$$




$$
\times\left\{\lambda^{2}\left|f^{\prime}\left(\frac{(2-\lambda) a+\lambda b}{2}\right)\right|+(1-\lambda)^{2}\left|f^{\prime}\left(\frac{(1-\lambda) a+(1+\lambda) b}{2}\right)\right|\right\},
$$

Corollary 25. Under the assumptions of Theorem 13 with $\theta=1$, we have the following Ostrowski type inequality

$$
\begin{gathered}
\left|f(x)-\frac{1}{b-a} \int_{a}^{b} f(u) d u\right| \\
\leq \frac{2^{\frac{s-1}{q}}}{(p+1)^{\frac{1}{p}}(b-a)}\left[(x-a)^{2}\left|f^{\prime}\left(\frac{x+a}{2}\right)\right|+(b-x)^{2}\left|f^{\prime}\left(\frac{x+b}{2}\right)\right|\right]
\end{gathered}
$$

for each $x \in[a, b]$.

Proof. For each $x \in[a, b]$, there exist $\lambda_{x} \in[0,1]$ such that $x=\left(1-\lambda_{x}\right) a+$ $\lambda_{x} b$. Hence we have $\lambda_{x}=\frac{x-a}{b-a}$ and $1-\lambda_{x}=\frac{b-x}{b-a}$. Therefore for each $x \in[a, b]$, from the inequality (20) we obtain the inequality (23).

Remark 6. We note that the inequality (23) is the same of the inequality in [2, Theorem 5].

Corollary 26. Under the assumptions of Theorem 21 with $\lambda=\frac{1}{2}$ and $\theta=0$, we have the following trapezoid type inequality

$$
\begin{aligned}
& \left|\frac{f(a)+f(b)}{2}-\frac{1}{b-a} \int_{a}^{b} f(x) d x\right| \\
\leq & \frac{b-a}{4}\left(\frac{1}{p+1}\right)^{\frac{1}{p}}\left(\frac{1}{2}\right)^{\frac{1-s}{q}}\left[\left|f^{\prime}\left(\frac{3 b+a}{4}\right)\right|+\left|f^{\prime}\left(\frac{3 a+b}{4}\right)\right|\right]
\end{aligned}
$$

which is the same of the inequality in [10, Theorem 8 (i)].

Corollary 27. Under the assumptions of Theorem 21 with $\lambda=\frac{1}{2}$ and $\theta=1$, we have the following midpoint type inequality

$$
\begin{aligned}
& \left|f\left(\frac{a+b}{2}\right)-\frac{1}{b-a} \int_{a}^{b} f(x) d x\right| \\
\leq & \frac{b-a}{4}\left(\frac{1}{p+1}\right)^{\frac{1}{p}}\left(\frac{1}{2}\right)^{\frac{1-s}{q}}\left[\left|f^{\prime}\left(\frac{3 b+a}{4}\right)\right|+\left|f^{\prime}\left(\frac{3 a+b}{4}\right)\right|\right]
\end{aligned}
$$

which is the same of the inequality in [10, Theorem 8 (ii)]. 
Corollary 28. Under the assumptions of Theorem 21 with $\lambda=\frac{1}{2}$ and $\theta=\frac{2}{3}$, we have the following trapezoid type inequality

$$
\begin{aligned}
& \left|\frac{1}{6}\left[f(a)+4 f\left(\frac{a+b}{2}\right)+f(b)\right]-\frac{1}{b-a} \int_{a}^{b} f(x) d x\right| \\
\leq & \frac{b-a}{12}\left(\frac{1+2^{p+1}}{3(p+1)}\right)^{\frac{1}{p}} 2^{\frac{s-1}{q}}\left[\left|f^{\prime}\left(\frac{3 b+a}{4}\right)\right|+\left|f^{\prime}\left(\frac{3 a+b}{4}\right)\right|\right] .
\end{aligned}
$$

Remark 7. If $\left|f^{\prime}\right|^{q}, q>1$, is concave on $[a, b]$, using the power mean inequality, we have

$$
\begin{aligned}
\left|f^{\prime}(\lambda x+(1-\lambda) y)\right|^{q} & \geq \lambda\left|f^{\prime}(x)\right|^{q}+(1-\lambda)\left|f^{\prime}(y)\right|^{q} \\
& \geq\left(\lambda\left|f^{\prime}(x)\right|+(1-\lambda)\left|f^{\prime}(y)\right|\right)^{q},
\end{aligned}
$$

$\forall x, y \in[a, b]$ and $\lambda \in[0,1]$. Hence

$$
\left|f^{\prime}(\lambda x+(1-\lambda) y)\right| \geq \lambda\left|f^{\prime}(x)\right|+(1-\lambda)\left|f^{\prime}(y)\right|
$$

so $\left|f^{\prime}\right|$ is also concave. Then by the inequality (1), we have

$$
\left|f^{\prime}\left(\frac{3 b+a}{4}\right)\right|+\left|f^{\prime}\left(\frac{3 a+b}{4}\right)\right| \leq 2\left|f^{\prime}\left(\frac{a+b}{2}\right)\right| .
$$

Thus, using the inequality (27) in (24), (25) and (26) we get

$$
\begin{aligned}
\left|\frac{f(a)+f(b)}{2}-\frac{1}{b-a} \int_{a}^{b} f(x) d x\right| & \leq \frac{b-a}{2}\left(\frac{1}{p+1}\right)^{\frac{1}{p}}\left(\frac{1}{2}\right)^{\frac{1-s}{q}}\left|f^{\prime}\left(\frac{a+b}{2}\right)\right|, \\
\left|f\left(\frac{a+b}{2}\right)-\frac{1}{b-a} \int_{a}^{b} f(x) d x\right| & \leq \frac{b-a}{2}\left(\frac{1}{p+1}\right)^{\frac{1}{p}}\left(\frac{1}{2}\right)^{\frac{1-s}{q}}\left|f^{\prime}\left(\frac{a+b}{2}\right)\right|,
\end{aligned}
$$

and

$$
\begin{aligned}
& \left|\frac{1}{6}\left[f(a)+4 f\left(\frac{a+b}{2}\right)+f(b)\right]-\frac{1}{b-a} \int_{a}^{b} f(x) d x\right| \\
\leq & \frac{b-a}{12}\left(\frac{1+2^{p+1}}{3(p+1)}\right)^{\frac{1}{p}}\left(\frac{1}{2}\right)^{\frac{1-s}{q}}\left|f^{\prime}\left(\frac{a+b}{2}\right)\right|,
\end{aligned}
$$

respectively, where $s \in(0,1]$ and $1 / p+1 / q=1$. 


\section{Some Applications for Special Means}

in [8], the following example is given.

Let $s \in(0,1)$ and $a, b, c \in \mathbb{R}$.We define function $f:[0, \infty) \rightarrow \mathbb{R}$ as

$$
f(t)=\left\{\begin{array}{cc}
a, & t=0 \\
b t^{s}+c, & t>0
\end{array} .\right.
$$

If $b \geq 0$ and $0 \leq c \leq a$, then $f \in K_{s}^{2}$. Hence, for $a=c=0, b=s+1$, $s \in\left(0, \frac{1}{q}\right), q \geq 1$, we have $f:[0, \infty) \rightarrow[0, \infty), f(t)=t^{s+1},\left|f^{\prime}\right|^{q} \in K_{s}^{2}$.

Let us recall the following special means of arbitrary real numbers $a, b$ with $a \neq b$ and $\alpha \in[0,1]$ :

1. The weighted arithmetic mean

$$
A_{\alpha}(a, b):=\alpha a+(1-\alpha) b, a, b \in \mathbb{R} .
$$

2. The unweighted arithmetic mean

$$
A(a, b):=\frac{a+b}{2}, a, b \in \mathbb{R} .
$$

3. The Logarithmic mean

$$
L(a, b):=\frac{b-a}{\ln b-\ln a}, \quad a \neq b, \quad a, b>0 .
$$

4. Then $p$-Logarithmic mean

$$
L_{p}(a, b):=\left(\frac{b^{p+1}-a^{p+1}}{(p+1)(b-a)}\right)^{\frac{1}{p}}, p \in \mathbb{R} \backslash\{-1,0\}, a, b>0 .
$$

Now, using the results of Section 2, some new inequalities are derived for the above means.

Proposition 29. Let $a, b \in \mathbb{R}$ with $0<a<b, q \geq 1, s \in\left(0, \frac{1}{q}\right)$ and $\lambda, \theta \in[0,1]$ we have the following inequality:

$$
\begin{aligned}
& \left|(1-\theta) A_{\lambda}\left(a^{s+1}, b^{s+1}\right)+\theta A_{\lambda}^{s+1}(a, b)-L_{s+1}^{s+1}(a, b)\right| \\
\leq & (b-a) A_{1}^{1-\frac{1}{q}}(\theta)(s+1)\left\{\lambda^{2}\left[a^{s q} A_{2}(\theta, s)+A_{\lambda}^{s q}(b, a) A_{3}(\theta, s)\right]^{\frac{1}{q}}\right. \\
& \left.+(1-\lambda)^{2}\left[b^{s q} A_{2}(\theta, s)+A_{\lambda}^{s q}(b, a) A_{3}(\theta, s)\right]^{\frac{1}{q}}\right\}
\end{aligned}
$$

where $A_{1}(\theta), A_{2}(\theta, s), A_{3}(\theta, s)$ are defined as in Theorem 4 . 
Proof. The assertion follows from Theorem 4 for the function $f(t)=t^{s+1}, t \in$ $[0, \infty), s \in\left(0, \frac{1}{q}\right)$.

Proposition 30. Let $a, b \in \mathbb{R}$ with $0<a<b, p, q>1, \frac{1}{p}+\frac{1}{q}=1$, $s \in\left(0, \frac{1}{q}\right)$ and $\lambda, \theta \in[0,1]$ we have the following inequality:

$$
\begin{aligned}
& \left|(1-\theta) A_{\lambda}\left(a^{s+1}, b^{s+1}\right)+\theta A_{\lambda}^{s+1}(a, b)-L_{s+1}^{s+1}(a, b)\right| \\
\leq & (b-a)\left(\frac{\theta^{p+1}+(1-\theta)^{p+1}}{p+1}\right)^{\frac{1}{p}}(s+1)^{1-\frac{1}{q}} \\
& \times\left[\lambda^{2}\left(a^{s q}+A_{\lambda}^{s q}(b, a)\right)^{\frac{1}{q}}+(1-\lambda)^{2}\left(b^{s q}+A_{\lambda}^{s q}(b, a)\right)^{\frac{1}{q}}\right] .
\end{aligned}
$$

where $1 / p+1 / q=1$.

Proof. The assertion follows from Theorem 13 for the function $f(t)=$ $t^{s+1}, t \in[0, \infty), s \in\left(0, \frac{1}{q}\right)$.

\section{References}

[1] M. Alomari, M. Darus, S.S. Dragomir, New inequalities of Simpson's Type for $s$-convex functions with applications, RGMIA Res. Rep. Coll., 12, No. 4 (2009), Article 9.

[2] M. Alomaria, M. Darus, S.S. Dragomir, P. Cerone, Ostrowski type inequalities for functions whose derivatives ares-convex in the second sense, Applied Mathematics Letters, 23 (2010), 1071-1076.

[3] M.W. Alomari, M. Darus, U.S. Kirmaci, Some inequalities of HermiteHadamard type for s-convex functions, Acta Math. Scientia, 31B, No. 4 (2011), 1643-1652.

[4] W.W. Breckner, Stetigkeitsaussagen für eine Klasse verallgemeinerter konvexer funktionen in topologischen linearen Räumen, Publ. Inst. Math., 23 (1978), 13-20.

[5] S.S. Dragomir, S. Fitzpatrik, The Hadamard's inequality for $s$-convex functions in the second sense, Demonstration Math., 32, No. 4 (1999), 687-696. 
[6] S.S. Dragomir, C.E.M. Pearce, Selected Topics on Hermite-Hadamard Inequalities and Applications, RGMIA Monographs, Victoria University (2000).

[7] S.S. Dragomir, Th.M. Rassias, Ostrowski Type Inequalities and Applications in Numerical Integration, Kluwer Academic Publishers, Dorcdrecht, Boston, London (2002).

[8] H. Hudzik, L. Maligranda, Some remarks on s-convex functions, Aequationes Math., 48 (1994), 100-111.

[9] U.S. Kirmaci, M.K. Bakula, M.E. Özdemir, J. Pečarić, Hadamard-type inequalities for s-convex functions, Applied Mathematics and Computation, 193 (2007), 26-35.

[10] J. Park, Hermite-Hadamard-type inequalities for real $\alpha$-star $s$-convex mappings, J. Appl. Math. \& Informatics, 28, No. 5-6 (2010), 1507-1518.

[11] M.Z. Sarikaya, N. Aktan, On the generalization of some integral inequalities and their applications, Mathematical and Computer Modelling, $\mathbf{5 4}$ (2011), 2175-2182.

[12] M.Z. Sarikaya, E. Set, M.E. Özdemir, On new inequalities of Simpson's type for s-convex functions, Computers and Mathematics with Applications, 60 (2010), 2191-2199.

[13] M.Z. Sarikaya, E. Set, M.E. Özdemir, On new inequalities of Simpson's type for convex functions, RGMIA Res. Rep. Coll., 13, No. 2 (2010), Article 2 . 